\title{
Both perceptual and conceptual factors influence taste-odor and taste-taste interactions
}

\author{
ROBERT A. FRANK and NICOLETTE J. VAN DER KLAAUW \\ University of Cincinnati, Cincinnati, Ohio \\ and \\ HENDRIK N. J. SCHIFFERSTEIN \\ Agricultural University, Wageningen, The Netherlands
}

\begin{abstract}
Observers are often asked to make intensity judgments for a sensory attribute of a stimulus that is embedded in a background of "irrelevant" stimulus dimensions. Under some circumstances, these background dimensions of the stimulus can influence intensity judgments for the target attribute. For example, judgments of sweetness can be influenced by the other taste or odor qualities of a solution (Frank \& Byram, 1988; Kamen et al., 1961). Experiments 1 and 2 assessed the influence of stimulus context, instructional set, and reference stimuli on cross-quality interactions in mixtures of chemosensory stimuli. Experiment 1 demonstrated that odor-induced changes in sweetness judgments were dramatically influenced when subjects rated multiple attributes of the stimulus as compared with when they judged sweetness alone. Several odorants enhanced sweetness when sweetness alone was judged, while sweetness was suppressed for these same stimuli when total-intensity ratings were broken down into ratings for the sweetness, saltiness, sourness, bitterness, and fruitiness of each solution. Experiment 2 demonstrated a similar pattern of results when bitterness was the target taste. In addition, Experiment 2 showed that the instructional effects applied to both taste-odor and taste-taste mixtures. It was concluded that the taste enhancement and suppression observed for taste-odor and taste-taste mixtures are influenced by (1) instructional sets which influence subjects' concepts of attribute categories, and (2) the perceptual similarities among the quality dimensions of the stimulus.
\end{abstract}

Many psychophysical experiments involve observers making judgments about the intensitive dimensions of a stimulus. These judgments are often made for stimuli which produce a complex pattern of sensory activation both within and across various sensory modalities. As a result, the experiences associated with these stimuli are usually multidimensional. Obviously, a request that an observer rate the intensity of such a complex stimulus can be ambiguous. For example, most foods and beverages consist of a multitude of chemical substances which are capable of stimulating olfactory, gustatory, tactile, temperature, and nociceptive receptors. An observer could rate the intensity of the taste, smell, "feel," or "tingle" associated with the stimulus, or could rate the intensity of different qualities within one modality (for example, sweet, salty, sour, or bitter for taste). It would also be

The research described in Experiments 1 and 2 was presented in preliminary form at the annual meeting of the Association for Chemoreception Sciences, Sarasota, Florida, April 1992. The collaboration of H. N. J. Schifferstein on the projects was supported by travel grants from the European Chemoreception Research Organization and the LEBFonds. In addition, the Netherlands Organization for Scientific Research (NWO) is acknowledged for supporting H. N. J. Schifferstein during the research period by means of a NWO PSYCHON grant (560-262032) to J. E. R. Frijters. Correspondence should be addressed to R. A. Frank, ML\#376, Department of Psychology, University of Cincinnati, Cincinnati, OH 45221-0376. possible for an observer to make estimates of the overall strength of the stimulus by integrating the component dimensions. Depending on the hypotheses under investigation, one or another of these approaches to assessing stimulus intensity should be employed. Sometimes the researcher's primary interest may be the total intensity of a stimulus, whereas at other times the research questions focus on the intensity of a particular stimulus component. To use an analogy from the concert hall, sometimes a conductor is concerned with the overall loudness of the entire orchestra whereas at other times the relative loudness of a particular instrumental section is of greater concern.

It seems likely that the principles that govern the integration of multiple stimulus dimensions into a single intensity estimate are different from those which govern intensity ratings for a single stimulus attribute in the face of a complex, multidimensional background. Anderson (1981) has studied the processes of integration using information-integration theory and functional measurement. Information-integration experiments typically attempt to discover how two or more independent variables combine to jointly influence a response along a common dimension. For example, the contribution of multiple taste qualities to total taste intensity has been studied by several investigators using the information-integration approach (Frank \& Archambo, 1986; Schifferstein \& Frijters, 1990). Other experiments have focused on judgments 
of a specific target taste in a mixture stimulus eliciting multiple taste qualities (Kamen, Pilgrim, Gutman, \& Kroll, 1961; Lawless, 1977, 1979). The problem of extracting and rating the strength of a specific stimulus attribute from a multidimensional stimulus background is the subject of the present report.

Our interests in how the "background" dimensions of a stimulus influence intensity judgments of a target quality began with investigations of odor-induced changes in taste perception. Frank and Byram (1988) and Shaffer and Frank (1990) had reported that odor-induced enhancement of taste was both odorant- and tastant-dependent. An odor (e.g., almond) which enhances one taste quality (e.g., sweetness) may suppress another (e.g., saltiness). Subsequent research provided some support for the hypothesis that the perceptual similarity between a taste and odor sensation is predictive of whether a specific taste quality is enhanced or suppressed in a taste-odor mixture (Frank, Shaffer, \& Smith, 1991). It has also been reported that the instructional set given to subjects can have a substantial impact on odor-induced enhancement of sweetness (Frank, Wessel, \& Shaffer, 1990). The latter finding suggests that cognitive factors related to the demands of the experimental task have an important influence on responses to taste-odor mixtures.

The two experiments described below assessed the influence of several sensory and cognitive factors on judgments of taste-odor and heterogeneous taste-quality mixtures. ${ }^{1}$ The goal of these experiments was to evaluate the influence of these factors on reports of taste-quality enhancement or suppression in these two types of mixture.

\section{EXPERIMENT 1}

Frank et al. (1990) reported that the sweetness-enhancing effect of strawberry odorant was dependent on the instructional set used by subjects. If subjects were asked to rate sweetness alone and to ignore all other attributes of the stimuli, the sweetness ratings of sucrose solutions were enhanced by strawberry odorant. This effect was observed at concentrations of sucrose that ranged from 0.1 to $0.5 \mathrm{M}$, and the effect increased as strawberry-odorant concentration increased from $0.25 \%$ to $1.0 \%$. (This finding replicated previous research conducted in our laboratory using fewer concentrations of the odorant [Frank \& Byram, 1988; Frank, Ducheny, \& Mize, 1989].) Much to our surprise, a completely different result was obtained when we allowed subjects to judge the same set of sucrose and strawberry solutions using multiple-attribute ratings rather than having them rate sweetness alone. When subjects judged the sweetness, saltiness, sourness, bitterness, and "other" tastes of the stimuli, no enhancement of the sweetness of sucrose was observed at any of the concentrations of strawberry odorant.

There are at least two possible explanations for the outcome of the Frank et al. (1990) experiment. One explanation focuses on the influence that instructions have on a person's "working concept" of a sensory attribute, and the extent to which a subject is encouraged or discouraged to characterize the various attributes of the stimuli as falling into a particular sensory category. Based on their experiences, people develop overlapping, but distinctive, concepts about what constitutes "sweet," "sour," "red," "cool," or "floral." When a person enters a laboratory, the experimenter attempts to direct the subject's attention toward a particular aspect of a multidimensional stimulus through the use of instructions and possibly through the presentation of prototypical stimuli. These procedures represent the experimenter's attempt to align the concepts of the subject with other subjects and with those of the experimenter. (See O'Mahony, 1991, for a discussion of concept alignment in sensory research.) It follows from the preceding discussion that the instructions and prototypes that are offered can influence what subjects will include in or exclude from their judgments of a particular sensory attribute. Instructions could bias a subject to be relatively lenient regarding what they were willing to call "sweet" by encouraging them to develop a more expansive concept of sweetness, or subjects could be encouraged to develop a very narrow concept of sweet which excluded a variety of sensory concepts which were perceptually similar to, but discriminable from, sweetness (e.g., fruitiness).

Stimulus context may also influence what is included in or excluded from a person's concept of a particular sensory attribute. For example, in an experiment which involves the judgment of sucrose and strawberry-flavored solutions and their mixtures, the restriction of the stimuli to solutions that are either sweet-tasting or strawberrysmelling (or both) limits the range of possible experiences in a way that may encourage subjects to make subtle distinctions among the stimuli so that attribute categories are used with near equal frequency. This prediction follows from Parducci's (1965) range-frequency model since it might apply to the range of stimulus qualities in addition to the range of stimulus intensities. Parducci noted that people tend to use all the points on a category scale regardless of the range of stimulus intensities. The subject's use of stimulus attributes may operate in a similar manner. That is, the subjects are biased toward using all the attribute categories irrespective of the range of qualities they experience in a set of stimuli. Lawless, Glatter, and Hohn (1991) identified this as the response-frequency-bias hypothesis in their work on context-dependent changes in the perception of odor quality. Frank et al. (1990) used a very restricted set of stimulus qualities (sweet and strawberry) with a broad set of possible attributes (sweet, salty, sour, bitter, "other'). This may have biased subjects toward using the categories of salty, sour, bitter, and "other" to describe the subtle differences among the stimuli even when these differences were minimal. Distributing taste intensity across the additional gustatory categories may have eliminated strawberry-induced enhancement of sweetness because the odorant's contribution to stimulus intensity was now divided among several target tastes.

The effect of stimulus context and the number of response categories on odor-induced changes in the sweet- 
ness of sucrose was evaluated in Experiment 1. Subjects judged sweetness in both a "narrow" stimulus context centered around fruit-like attributes (sweet and sour tastes with fruity odors) and a "broad" context consisting of a more diverse set of tastes and odors. These two stimulus contexts were combined factorially with instructional conditions that ranged from judging sweetness only to judging the total intensity of the stimuli plus six other sensory attributes.

\section{Method}

\section{Subjects}

The subjects were 20 volunteers, 6 males and 14 females, recruited from the student population at the University of Cincinnati. The subjects were naive as to the purpose of the experiment and were paid for their participation.

\section{Stimuli}

The stimuli consisted of aqueous solutions made with distilled water. Four tastants were used: $0.25 \mathrm{M}$ sucrose (Fisher S-5), $2.5 \mathrm{mM}$ citric acid (Sigma C-0759), 0.14 M NaCl (Fisher S-271), $0.15 \mathrm{mM}$ quinine hemisulfate (Sigma Q-1250). The five odorants were $1.0 \% \mathrm{v} / \mathrm{v}$ strawberry (F\&C International 076201), $0.4 \% \mathrm{v} / \mathrm{v}$ lemon (Alex Fries 66-894), 0.6\% v/v almond (Alex Fries 66-794), $0.2 \% \mathrm{v} / \mathrm{v}$ wintergreen (Alex Fries), and $0.5 \% \mathrm{v} / \mathrm{v}$ chocolate (Alex Fries 81-056). The remaining stimuli included all possible mixtures of the odorants with the tastants. Concentrations were chosen so that the total intensities of the odorants were approximately matched. The total intensities of the tastants were also approximately matched. All solutions were made at least $24 \mathrm{~h}$ prior to testing and refrigerated between days of testing. New solutions were prepared every 7 days. The mixtures were made by combining the solute and odorant concentrate, and then bringing the solution up to volume. Stimulus volume was set at $2.5 \mathrm{ml}$, and the solutions were always sampled at room temperature. Previous research (Frank \& Byram, 1988; Shaffer \& Frank, 1990) and pilot studies had shown that the flavors produce no taste sensations at these concentrations (as demonstrated by rating the odorant solutions with the nostrils pinched).

\section{Procedure}

Prior to their participation in the experiment, the subjects were given a brief orientation session and test. They were given the unmixed solutions of sucrose, sodium chloride, citric acid, and quinine sulfate and told that these stimuli represented examples of sweet, salty, sour, and bitter, respectively. They were then given three concentrations of sucrose $(0.125,0.25$, and $0.5 \mathrm{M})$ and a distilled water stimulus and asked to rate each of these for sweetness. If the ratings did not increase from distilled water to $0.5 \mathrm{M}$ sucrose as a monotonic function of sucrose concentration, the subject was excused. Next, the subjects sampled three concentrations of citric acid $(1.25,2.5$, and $5 \mathrm{mM})$ plus distilled water and were asked to rate sourness. If the ratings were not a monotonic increasing function of citric acid concentration, the subject was excused.

In the experimental conditions, the subjects judged the sensory attributes of two different sets of stimuli using three different sets of instructions. The two sets of stimuli were designated the narrow and the broad stimulus contexts. The narrow stimulus context consisted of the following stimuli: distilled water, sucrose, citric acid, strawberry, lemon, almond, and all possible mixtures of the odorants with the tastants. The stimuli in this set were always judged twice by the subjects (in two blocks of trials), so that an experimental session with this set of stimuli consisted of 24 trials. The broad stimulus context consisted of distilled water, sucrose, citric acid, sodium chloride, quinine sulfate, strawberry, lemon, wintergreen, chocolate, and all possible mixtures of the tastants and odorants. One judgment was made for each stimulus for a total of 25 trials per session. The subjects participated in two identical testing sessions using the broad context stimuli so that each stimulus was judged twice.

Three different sets of instructions were factorially combined with the two stimulus contexts. In one condition, the subjects were instructed to judge the sweetness of the stimuli. In a second condition, the subjects were to judge the sweetness, sourness, and fruitiness of the solutions. In a third condition, the subjects first rated the total intensity of the stimuli; this was then broken down into ratings of sweetness, saltiness, sourness, bitterness, fruitiness, and "other" taste of the solutions. When appropriate, a subject was provided with a reference sample which defined the target sensation to be rated on each scale. The sweetness reference was $0.25 \mathrm{M}$ sucrose, the saltiness reference was $0.14 \mathrm{M}$ sodium chloride, the sourness reference was $2.5 \mathrm{mM}$ citric acid, the bitterness reference was $0.15 \mathrm{mM}$ quinine hemisulfate, and the fruitiness reference was $1 \% \mathrm{v} / \mathrm{v}$ strawberry odorant. All the stimulus attributes were rated on a 21-point category scale labeled 0-no taste, 5-weak, 10-medium. 15-strong, and 20-very strong.

All subjects experienced the six factorial combinations of stimulus context and instructions in the same order over nine sessions, separated by at least $24 \mathrm{~h}$. The subjects first completed the condition involving the narrow stimulus context and ratings of sweetness only, then completed sweetness-only ratings for the broad stimulus context in two consecutive sessions. Next, they rated the narrow context stimuli, using ratings of sweet, sour, and fruity, followed by two sessions with the same instructions and the broad stimulus context. Finally, they rated the narrow context stimuli in the condition where total intensity and six other attributes were evaluated. These instructions were then used for two sessions with the broad context stimuli. This sequence of conditions was used so that subjects would move from simpler to more complex tasks as they completed each condition of the experiment.

Under all conditions, the samples were presented in plastic medicine cups. The subjects were instructed to take all of the solution into their mouths and swallow it, make the appropriate rating(s), rinse with distilled water, and then wait for $30 \mathrm{sec}$ before sampling the next stimulus. The stimuli were always presented in random order.

\section{Results}

Since every stimulus was rated twice for each condition, the arithmetic mean of the ratings was calculated for each subject under all six conditions. These means were used for statistical analyses.

The sweetness of sucrose, citric acid, strawberry odor, lemon odor, and their mixtures were rated under all six experimental conditions. The mean sweetness ratings collapsed across sucrose and mixtures of sucrose with strawberry or lemon are shown in Figure 1 plotted as a function of the three sets of instructions and the two stimulus contexts. This figure shows that the sweetness ratings decreased when the number of judged attributes increased. The absolute ratings for sweetness were highest when only sweetness was rated and lowest when total-intensity ratings were broken down. A $2 \times 3 \times 3$ (context $\times$ instructions $\times$ mixture type) repeated measures analysis of variance (ANOVA) confirmed that instructions significantly affected sweetness ratings $[F(2,38)=25.55, p<.001]$. In addition, subjects gave slightly, but significantly, higher sweetness ratings in the broad stimulus context $[F(1,19)$ $=5.77, p<.05]$.

The sweetness ratings for sucrose, the sucrosestrawberry mixture, and the sucrose-lemon mixture were 


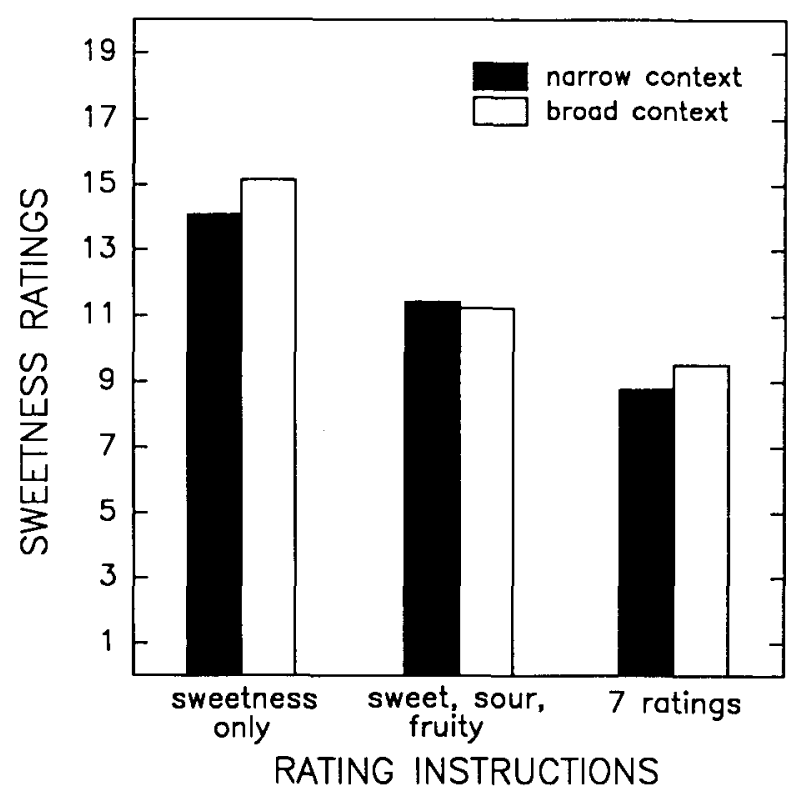

Figure 1. Mean sweetness ratings collapsed across sucrose and mixtures of sucrose with strawberry or lemon for the three instructional sets and two stimulus contexts. differentially influenced by the instructional set, as indicated by a significant instructions $\times$ mixture type (sucrose alone or mixed with strawberry or lemon) interaction $[F(4,76)=5.03, p<.001]$. In Figure 2, odor-induced changes in the sweetness of sucrose are plotted for mixtures of sucrose with strawberry or lemon. It is clear from Figure 2A that adding strawberry odor to sucrose enhanced the sweetness when subjects rated sweetness only. However, the sweetness was virtually unaffected when the task was to rate sweetness, sourness, and fruitiness on separate scales. Strawberry odor suppressed the sweetness of sucrose when subjects rated total intensity and broke that down into six categories. The pattern was the same for the broad and narrow stimulus context. A $2 \times 3 \times 2$ (context $\times$ instructions $\times$ mixture type) repeated measures ANOVA for the sucrose-strawberry data confirmed that the instructions $\times$ mixture type (sucrose alone or mixed with strawberry) interaction was significant $[F(2,38)=9.46, p<.001]$. This indicates that the effect of strawberry odor on the sweetness of sucrose was instruction-dependent. This was not the case for lemon odor. Figure 2B illustrates the effects of lemon odor on the sweetness of sucrose. For the sucrose-lemon data, the instructions $\times$ mixture type interaction was not significant $[F(2,38)=1.69, p>.05]$.

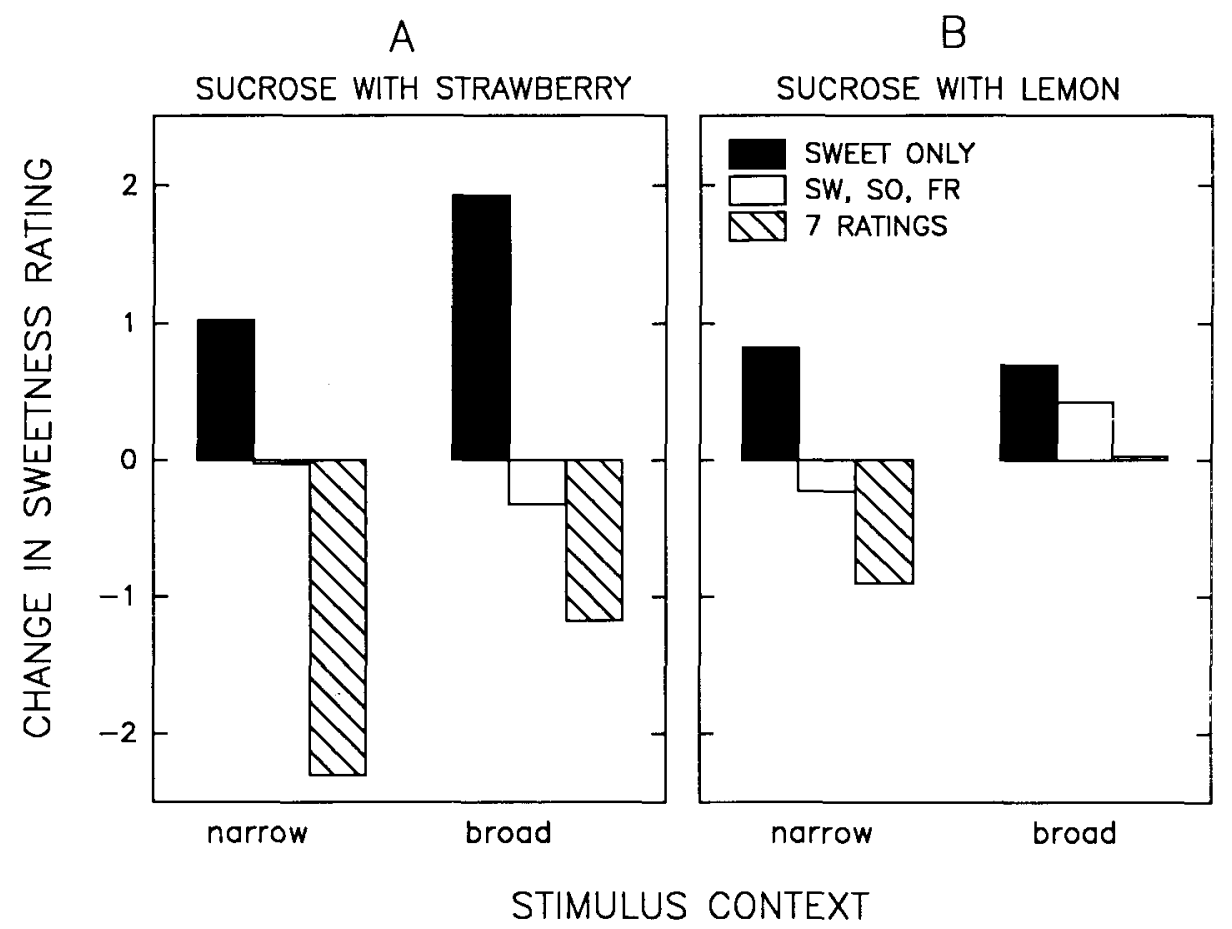

Figure 2. Strawberry and lemon odorant-induced changes in the sweetness ratings of sucrose as a function of instructional set and stimulus context. The ratings shown in the figure are differences in sweetness from unmixed sucrose. 


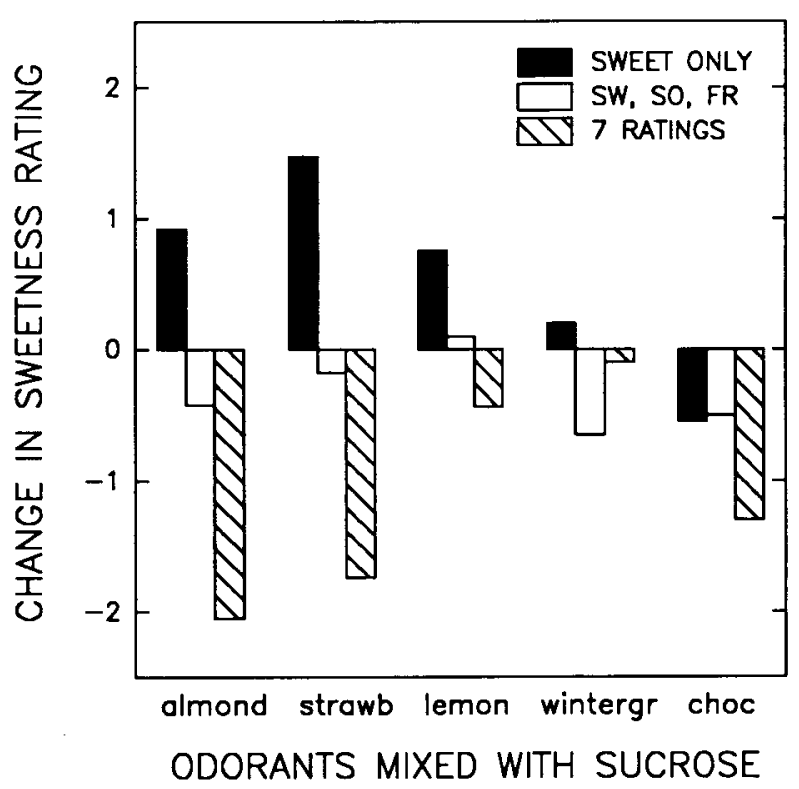

Figure 3. Odor-induced changes in the sweetness of sucrose for all odorant-sucrose combinations collapsed across instructional set and stimulus context. The ratings shown in the figure are differences in sweetness from unmixed sucrose.

In Figure 3, the odor-induced changes in sweetness of sucrose are plotted for all of the odorants. The data for almond are from the three instructional conditions in the narrow context only. For wintergreen and chocolate, the means are for each of the instructional conditions in the broad stimulus context only. The means for strawberry and lemon represent the averages over both the narrow and the broad context. Using a $3 \times 2$ repeated measures ANOVA to evaluate the changes in sweetness rating induced by each of the odorants, a significant instructions $X$ mixture type interaction was found for strawberry (as noted above) and for almond $[F(2,38)=5.34, p<.001]$. Notice that the changes in sweetness induced by almond and strawberry odorant were similar across the instructional conditions.

The sourness of citric acid seems much less affected by manipulations of stimulus context and instructions. In Figure 4, the mean sourness ratings collapsed over citric acid alone and citric acid with strawberry or lemon odor are plotted as a function of instructional set and stimulus context. Repeated measures ANOVAs revealed no significant effects of context or instructions. In addition, repeated measures $t$ tests revealed only one odor-induced change in the sourness of citric acid. Chocolate odor suppressed the sourness of citric acid when subjects made total-intensity judgments that were broken down into component ratings [mean sourness of unmixed citric acid = 7.3 , while the mean sourness of a chocolate-citric acid mixture was $4.8 ; t(19)=2.4, p<.05$ ].

\section{Discussion}

One of the goals of Experiment 1 was to determine whether stimulus context or instructional set was responsible for the changes in strawberry odor's effects on the sweetness of sucrose, as reported by Frank et al. (1990). The results clearly show that instructional set was the more important variable (see Figure 2).

The effects of instructions on odor-induced changes in sweetness ratings may be related to the ability of the instructions and reference stimuli to influence the subjects' concept of sweetness. When they were judging only the sweetness of the stimuli, the subjects sampled a sucrose solution and were told that it represented an example of sweetness. Examples of stimuli that were not sweet were not provided. This may have biased the subjects toward a rather broad concept of sweetness that included dimensions of stimuli that were either perceptually similar to sweetness or often associated with sweet-tasting stimuli. An assessment of the fruity ratings from the total-intensity instructional condition provides some support for this hypothesis. As shown in Figure 5, the ability of the odorants to add to the fruity ratings of sucrose in the total-intensity condition was positively and highly correlated with their ability to enhance sweetness in the sweetness-only condition. When sweet, sour, and fruity were rated separately, odor-induced enhancement of sweetness disappeared. This suggests that sweetness and fruitiness were combined when the subjects rated only the sweetness of the stimuli. The observation that sweetness ratings were

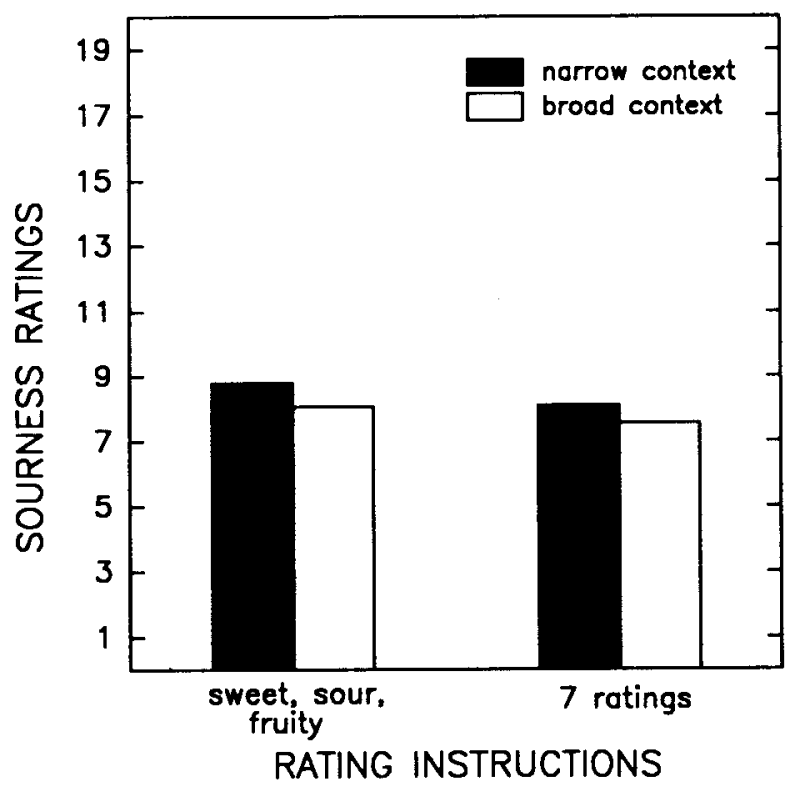

Figure 4. Mean sourness ratings for citric acid collapsed across citric acid alone and citric acid mixed with strawberry or lemon odor plotted as a function of instructional set and stimulus context. 


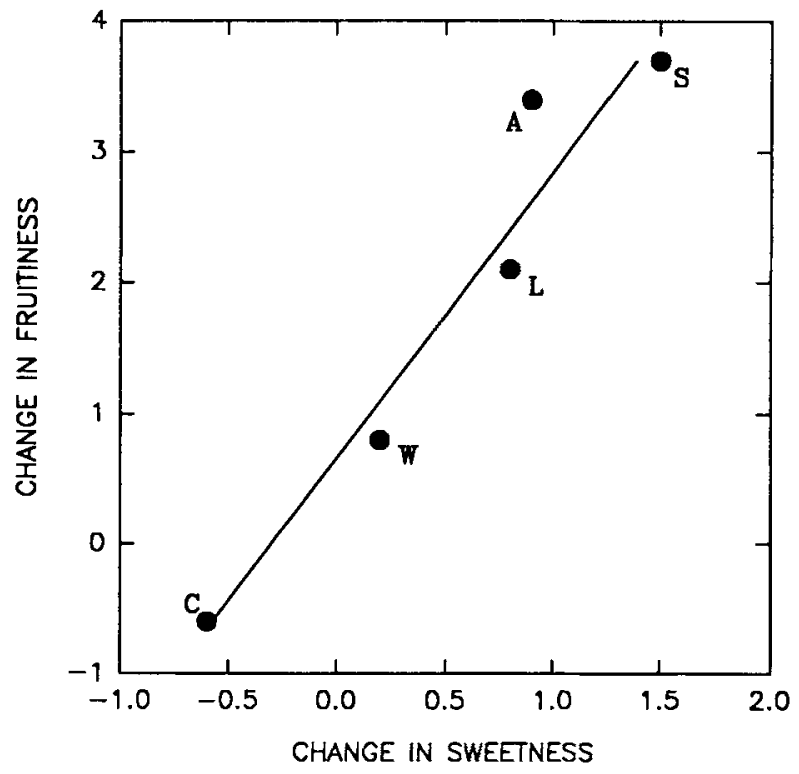

Figure 5. The relationship between odor-induced changes in fruitiness of sucrose (in the total-intensity-rating condition) and odorinduced changes in the sweetness of sucrose (in the sweetness-only rating condition). The ratings shown in the figure are differences in sweetness and fruitiness ratings between the sucrose-odorant mixture and unmixed sucrose. The data points have been fit to the linear function in the figure using a least squares criterion. $S=$ strawberry, $\mathrm{A}=$ almond, $\mathrm{L}=$ lemon, $\mathrm{W}=$ wintergreen, $\mathrm{C}=$ chocolate.

highest in the sweetness-only rating condition supports this view.

On the basis of our previous research and the results of Experiment 1, it is hypothesized that two variables contribute to odor-induced enhancement of taste intensity: the perceptual similarity of the tastant and odorant and the availability of appropriate response categories. When an odorant elicits sensations that are perceptually similar to a target taste and when only one rating category is available, subjects will tend to combine the taste and odor dimensions, leading to higher intensity ratings for the target taste. When the taste and odor sensations are dissimilar or when appropriate additional attribute scales are provided, odor-induced taste enhancement will not be observed.

Frank et al. (1991) had subjects make similarity ratings for pairs of unmixed odorants and tastants. For the odorants used in Experiment 1, they found that strawberry odorant was rated as most similar to sucrose, followed by almond, wintergreen, lemon, and chocolate. An examination of the sweetness-only condition in Figure 3 reveals that the similarity of the odorants to sucrose predicted the ability of the odorants to enhance the sweetness of sucrose reasonably well. It is also noteworthy that citric acid and lemon were rated to be just as similar as sucrose and strawberry. However, since there was no sournessonly rating condition in Experiment 1, there was no opportunity to observe lemon-induced enhancement of sourness. Two odorants (strawberry and almond) significantly suppressed sweetness in the total-intensity, six-attribute rating condition. Careful examination of the pattern of ratings across all the taste-odor mixtures revealed that odorinduced taste suppression occurred when the odorant significantly enhanced a "side" attribute of the mixture. For example, in the case of sweetness suppression for sucrose, strawberry and almond added significantly to the fruitiness of the strawberry-sucrose and almond-sucrose mixtures. In the only other instance of significant odorinduced taste suppression, chocolate added significantly to the bitterness of citric acid while at the same time suppressing sourness. Odor-induced taste suppression may be a result of dividing the total-intensity ratings among the relevant quality categories. Placing part of the total intensity into one category constrains what can be placed in another category, since the intensities of the components must sum to total intensity. Therefore, as mixture quality becomes more complex, and as additional, mixtureappropriate categories are provided to the subject, less of the total intensity is available for assignment to each category. Since the almond-sucrose and strawberrysucrose stimuli are both fruity and sweet, subjects reduced the sweet ratings of these mixtures to increase the fruity ratings in the total-intensity condition. However, neither strawberry nor almond reduced the sweetness of sucrose in the sweet, sour, fruity rating condition, because the rating given to a stimulus on one scale in no way constrained the ratings given on other scales.

Gillan (1983) assessed mixtures of two tastants (sucrose and sodium chloride) and two odorants (citral and anethole) using a procedure in which subjects made independent magnitude estimates of sweetness, saltiness, lemon, and licorice flavor. He reported that the odorants suppressed both the sweetness and the saltiness of the tastant-odorant mixtures. According to the hypothesis presented in the preceding paragraph, no taste suppression should have been observed because the ratings of the subjects in Gillan's study were not constrained by estimates of total intensity. However, it is somewhat difficult to determine the relevance of Gillan's findings to those of the present study because Gillan had subjects sniff the olfactory stimulus, sip the tastant, spit, and then make six attribute ratings. One might expect less integration of olfactory and gustatory stimulation under these conditions than under those used in the present study, in which subjects swallowed an aqueous solution containing both an odorant and a tastant. The perceived temporal and spatial coherence of taste and odor sensations may have an important impact on the interaction of these two modalities.

\section{EXPERIMENT 2}

It was hypothesized that the odor-induced enhancement of sweetness observed in Experiment 1 was related to the subjects' combining sweetness and fruitiness in the sweetness-only rating condition. In Experiment 2, the ability of reference stimuli to reduce the tendency to combine attributes, and thereby eliminate odor-induced en- 
hancement of sweetness, was evaluated. In Experiment 1, reference stimuli were used to help subjects understand what the experimenters meant by such terms as sweet, sour, or fruity. More reference stimuli were used as subjects rated more stimulus qualities. Therefore, the number of reference stimuli was confounded with instructional set. The number of reference stimuli and rating categories were manipulated independently in Experiment 2.

Experiment 2 also assessed whether the effects that were observed for taste-odor mixtures in Experiment 1 would be observed for heterogeneous taste mixtures, that is, for mixtures of tastants with different taste qualities. Experiment $2 \mathrm{~A}$ reexamined taste-odor mixtures; Experiment 2B evaluated several heterogeneous taste mixtures.

The generality of the findings of Experiment 1 was evaluated by using a different target taste quality in Experiment 2 . Previous research had focused on mixtureinduced changes in the sweetness of sucrose. Changes in the bitterness of quinine were evaluated in Experiment 2.

Finally, the perceptual similarities among the components in a mixture may have been important contributors to the pattern of results observed in Experiment 1. Therefore, an attempt was made to manipulate the similarity of the mixture components in Experiment 2. Sucrose and citric acid were chosen to mix with quinine in the heterogeneous taste-mixture experiment because quinine is rated as being much more similar to citric acid than to sucrose (Frank et al., 1991). It has also been found that the sourness of citric acid and bitterness of quinine are often confused (Gregson \& Baker, 1973; O'Mahony, Goldenberg, Stedmon, \& Alford, 1979). In addition, it has been reported that sucrose suppresses bitterness (Kamen et al., 1961; Lawless, 1977, 1979), whereas citric acid enhances it (Kamen et al., 1961). It was predicted that citric acid would produce enhancement of bitterness in the bitterness-only rating condition, and that this effect would disappear when multiple attribute judgments (including sour) were made. It was also predicted that sucrose-induced changes in bitterness would not be affected by instructional manipulations since sucrose and quinine are perceptually dissimilar.

Fewer data are available concerning the similarity between quinine and various odorants. However, we had collected similarity judgments for some odorant-tastant pairs (Frank et al., 1991), and on that basis selected lemon and almond odorants to be mixed with quinine. Since almond odor was rated as less similar to quinine than was lemon odor, it was predicted that lemon-induced changes in bitterness would demonstrate greater sensitivity to instructional manipulations than would almond-induced changes.

\section{Experiment 2A}

\section{Method}

Subjects. The subjects were 36 volunteers, 14 males and 22 females, recruited from the student population of the University of Cincinnati. Half of them were assigned at random to an experimental group that received all three conditions. The remaining 18 subjects were assigned to the control group and participated in only one con- dition (see below). The subjects were naive as to the purpose of the experiment, and were paid for their participation.

Stimuli. The stimuli consisted of aqueous solutions of reagent grade quinine hydrochloride (QHCl, Sigma Q-1125) at three concentration levels: 30,60 , and $120 \mu \mathrm{M}$. Two odorants were used$0.4 \% \mathrm{v} / \mathrm{v}$ lemon odor (Alex Fries, 66-894) and $0.6 \% \mathrm{v} / \mathrm{v}$ almond odor (Alex Fries 66-794)-and the mixtures of the three concentrations of $\mathrm{QHCl}$ were used with each of the odorants. All solutions were made with distilled water at least $24 \mathrm{~h}$ prior to testing, and were refrigerated between days of testing. New solutions were prepared every 7 days. The mixtures were made by combining the solute and odorant concentrate, and then bringing the solution up to volume. Stimulus volume was set at $2.5 \mathrm{ml}$, and the solutions were always sampled at room temperature.

Procedure. The 18 subjects in the experimental group were first instructed to rate only the bitterness of the solutions. One reference solution $(60 \mu \mathrm{M} \mathrm{QHCl})$ was presented to the subjects prior to testing. They were told that it represented a medium bitter stimulus on a 21-point scale labeled 0-no taste, 5-weak, 10-medium, 15-strong, 20-very strong. The instructions for the next session were identical, except that the subjects were presented with six more reference stimuli labeled sweet $(0.25 \mathrm{M}$ sucrose), salty $(0.14 \mathrm{M}$ sodium chloride), sour ( $2.5 \mathrm{mM}$ citric acid), lemon $(0.4 \% \mathrm{v} / \mathrm{v}$ lemon odorant), almond ( $0.6 \%$ almond odorant), and fruity (1.0\% strawberry odorant). They were instructed to exclude these qualities from their judgments of bitterness.

The last session required subjects to rate the total intensity of the solutions on the 21-point scale, and to divide this rating into estimates of the sweetness, saltiness, sourness, bitterness, almondness, lemonness, fruitiness, and "other" sensations, which were defined by the reference stimuli. The subjects who had completed the two previous conditions of the experiment judged the stimuli using the total-intensity instructions; the other 18 subjects participated only in the total-intensity condition. These 18 new subjects served as a control group for the effects of judging the stimuli in the two previous experimental conditions (Conditions A and B of Figure 6).

All stimuli were rated twice in each session in two blocks of trials. The samples were presented in plastic medicine cups. The subjects were instructed to take all of the solution into their mouths and swallow it, to make the appropriate rating(s), to rinse with distilled water, and then to wait for $30 \mathrm{sec}$ before sampling the next stimulus. The stimuli were always presented in random order.

\section{Results and Discussion}

The mean intensity ratings were calculated for the two judgments given to each stimulus in each of the experimental conditions. These means were used as raw data for all statistical analyses. The mean bitterness ratings of the stimuli in the four experimental conditions are shown in Figure 6. Panel A shows the bitterness ratings when subjects were given one reference stimulus and rated only the bitterness of the stimuli. Panel B shows bitterness ratings when only bitterness was rated, but seven reference stimuli were provided. Panel $\mathrm{C}$ shows bitterness ratings when subjects were given seven reference stimuli and instructed to rate total intensity, and then to break their ratings down into estimates of sweet, salty, sour, bitter, lemon, almond, and other. Panel D shows the bitterness ratings for the control group that participated in the totalintensity condition.

A $3 \times 3 \times 3$ (instructional set $\times$ mixture type $\times$ concentration) repeated measures ANOVA comparing the conditions shown in Panels A, B, and C of Figure 6 revealed 

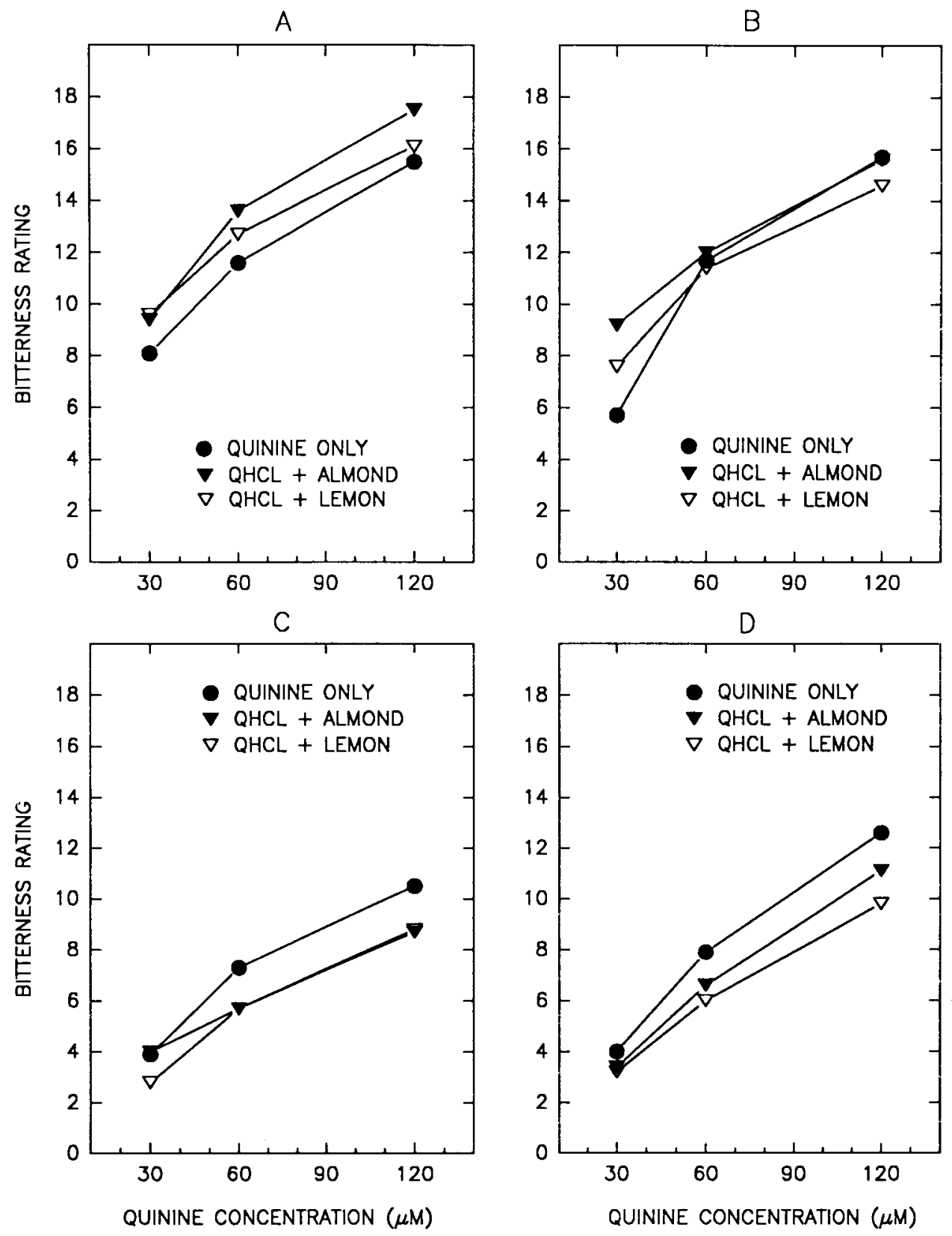

Figure 6. The effect of almond and lemon odorant on the bitterness of quinine across the four experimental conditions of Experiment $2 \mathrm{~A}$. Condition A: Bitterness ratings only and one reference stimulus (quinine). Condition B: Bitterness ratings only with seven reference stimuli. Condition C: Total-intensity ratings broken down into eight component ratings and seven reference stimuli provided. Condition D: Identical to Condition $C$ except that subjects in Condition $D$ did not participate in Conditions $A$ and $B$.

that quinine concentration had a significant effect on bitterness judgments $[F(2,34)=121.6, p<.001]$. The decreases in the ratings of bitterness that are shown in Figure 6 as one moves from Condition $\mathrm{A}$ to Condition $\mathrm{C}$ were also significant $[F(2,34)=39.37, p<.001]$. The only other significant effect was the interaction between instructional set and mixture type $[F(4,68)=6.49, p<.001]$.

The interactive effects of instructions and mixture type were further explored using $2 \times 3$ (quinine alone vs. qui- nine plus odorant $\times$ concentration) repeated measures ANOVAs to assess the effects of the odorants on the bitterness of quinine in each of the four experimental conditions shown in Figure 6. Separate ANOVAs were used to determine whether each odorant significantly changed the bitterness of the quinine stimuli. Quinine concentration produced a significant main effect in each of these analyses. The effects of the odorants on bitterness judgments for each of the experimental conditions are sum- 
Table 1

Changes in the Bitterness Judgments of Quinine Induced by the Tastants and Odorants Used in Experiment 2

\begin{tabular}{cccccc}
\multirow{2}{*}{$\begin{array}{c}\text { Experimental } \\
\text { Condition }\end{array}$} & \multicolumn{2}{c}{ Odorant } & & \multicolumn{2}{c}{ Tastant } \\
\cline { 2 - 5 } \cline { 4 - 6 } & Lemon & Almond & & Citric & Sucrose \\
\hline A & $=$ & + & + & - \\
B & $=$ & + & + & - \\
C & - & - & & - \\
D & - & $=$ & - & - \\
\hline
\end{tabular}

Note-Significant enhancement ( + ) and suppression $(-)$ of bitterness were demonstrated with ANOVAs (all $p<.05$ ). The $=$ symbol indicates no significant tastant or odorant-induced change in bittermess. Condition A: Bitterness ratings only and one reference stimulus (quinine). Condition B: Bitterness ratings only with seven reference stimuli. Condition $\mathrm{C}$ : Total-intensity ratings broken down into eight component ratings and seven reference stimuli provided. Condition D: Identical to Condition $\mathrm{C}$ except that subjects in Condition $\mathrm{D}$ did not participate in Conditions $\mathrm{A}$ and $\mathrm{B}$.

marized in Table 1. Notice that almond odor enhanced bitterness in Conditions A and B, but suppressed bitterness in Condition $C$. Although bitterness was reduced by almond odor at all three concentration levels in Condition $\mathrm{D}$, the effect was not significant at the .05 alpha level $(p<.12)$. Lemon odor suppressed bitterness in Conditions $C$ and $D$, had no effect on bitterness in Condition $B$, and just missed significantly enhancing bitterness in Condition $\mathrm{A}(p<.07)$.

The general pattern of results for the odor-induced changes in bitterness judgments was very similar to that observed for the odor-induced changes in sweetness judgments (see Experiment 1). This finding supports the conclusion that changes in instructions can influence an odorant's effects on estimates of taste' intensity. By contrast, manipulating the number of reference stimuli had little to no effect on odor-induced changes in bitterness (compare the odorant effects of Conditions A and B in Table 1). Therefore, it seems likely that differences in the number of reference stimuli were not responsible for the pattern of results observed in Experiment 1. Experiment $2 \mathrm{~A}$ also demonstrated that the instructional effects noted in Experiment 1 could be demonstrated with a different target taste (bitterness), and that the odor-induced changes in bitterness were not dependent on the concentration of quinine.

It had been predicted that lemon odor would induce a greater enhancement of bitterness than almond in the bitterness-only condition because lemon and quinine were rated as more similar than quinine and almond (Frank et al., 1991). However, the effects of lemon and almond on bitterness were not significantly different in the bitterness-only condition $[F(1,17)=2.29, p>.05]$. This finding may be interpreted in several ways: (1) Mixture component similarity may not play an important role in odor-taste interactions. (2) The nature of the similarity between the stimuli may not have been adequately measured by simply asking subjects to rate the similarity of the unmixed stimuli as was done by Frank et al. (1991). Perhaps similarity along a critical dimension (e.g., pleasantness) is important, not similarity "in general." Alternatively, similarity of the quality components in the mixture may be a better predictor of mixture interactions than similarity of the unmixed stimuli. (3) Even though lemon was rated as more similar to quinine than was almond, the difference between the two odorants regarding their similarity to quinine was small. On a scale that ranged from 1 (similar) to 9 (dissimilar), almond's similarity to quinine was rated at 7.25 and lemon's similarity to quinine was rated at 6.25 . Perhaps the difference between almond and lemon is not sufficient to produce a differential effect on the bitterness of quinine. It may be necessary to undertake additional research using more dissimilar odorants so that the effects of similarity can be more adequately assessed.

\section{Experiment 2B}

Most chemosensory-mixture research has focused on mixtures of tastants and the commonly reported phenomenon of mixture suppression (Bartoshuk, 1975). It was therefore of interest to determine whether the instructional effects observed with taste-odor mixtures would also apply to heterogeneous taste-quality mixtures. Experiment $2 B$ evaluated this question using the same design as Experiment 2A. However, sucrose-quinine and citric acid-quinine mixtures replaced the taste-odor mixtures as stimuli. The differences between Experiments $2 \mathrm{~A}$ and $2 \mathrm{~B}$ are described in the method section below.

\section{Method}

Subjects. The subjects were 40 volunteers, 17 males and 23 females, recruited from the student population of the University of Cincinnati. Half of them were assigned at random to the experimental group, and the remaining 20 were assigned to the control group.

Stimuli. The stimuli consisted of aqueous solutions of 30,60 , and $120 \mu \mathrm{M} \mathrm{QHCl}, 0.25 \mathrm{M}$ sucrose (Fisher S-5), and $2.5 \mathrm{mM}$ citric acid (Sigma Q-1250), and all possible combinations of quinine with sucrose and citric acid. The concentrations of sucrose and citric acid were chosen to approximately match the total intensity of the intermediate quinine solution.

Procedure. The procedures were identical to those in Experiment 2A.

\section{Results and Discussion}

The data were analyzed as described for Experiment 2A. The mean bitterness judgments for each stimulus and condition are shown in Figure 7. A $3 \times 3 \times 3$ repeated measures ANOVA was performed as in Experiment $2 \mathrm{~A}$, and it was found that both quinine concentration and instructional set had a significant effect on bitterness ratings $[F(2,38)=106.97, p<.001$, and $F(2,38)$ $=105.33, p<.001$, respectively]. The instructions $\times$ mixture type interaction was also significant $[F(4,76)=$ $4.41, p<.005]$.

As in Experiment $2 \mathrm{~A}, 2 \times 3$ ANOVAs were used to evaluate the mixture effects in each of the conditions (A through D) shown in Figure 6. The results of these analyses regarding the effects of citric acid and sucrose on the bitterness ratings of quinine are summarized in $\mathrm{Ta}$ ble 1 . Sucrose produced a systematic and substantial de- 

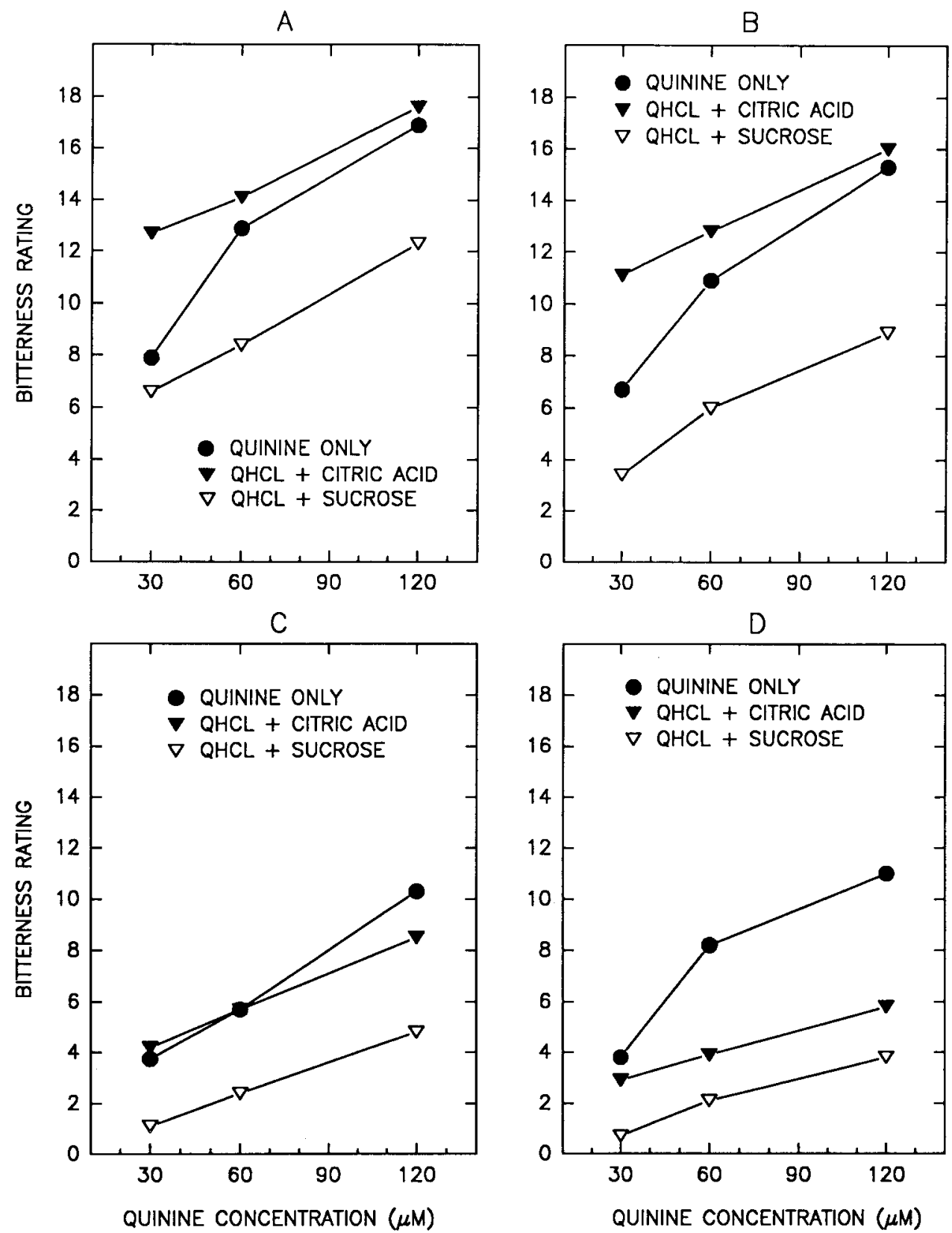

Figure 7. The effect of sucrose and citric acid on the bitterness of quinine across the four experimental conditions of Experiment 2B. Condition A: Bitterness ratings only and one reference stimulus (quinine). Condition B: Bitterness ratings only with seven reference stimuli. Condition C: Total-intensity ratings broken down into eight component ratings and seven reference stimuli provided. Condition D: Identical to Condition $\mathbf{C}$ except that subjects in Condition $D$ did not participate in Conditions $\mathbf{A}$ and $B$.

crease in the bitterness of quinine across all of the experimental conditions. However, citric acid's effects on bitterness resembled those of the odorants, that is, enhancement in the bitterness-only condition and suppression in the total-intensity condition.

Several conclusions can be drawn from the results of Experiment 2B. The effects of instructional set were very similar for both taste-odor and citric acid-quinine mixtures, suggesting that the factors that are responsible for the instructional effects are rather general, and probably apply to the judgment of many complex chemosensory stimuli.

The differential effect of citric acid and sucrose on the bitterness judgments across the instructional conditions confirms the prediction that the similarity of the mixture components may have an important influence on the ratings of particular stimulus attributes. It is hypothesized that citric acid and quinine exhibited the pattern of inter- 
action seen in Experiment $2 \mathrm{~B}$ because sour and bitter sensations are perceptually and/or conceptually similar. The nature of the similarity will require additional study, but one dimension of potential importance is the hedonic dimension. Perhaps the unpleasantness of citric acid adds to the unpleasantness of quinine, thus contributing to an overall increase in the unpleasantness of the mixture. This increase in unpleasantness may then be reported as an increase in bitterness by the subjects, since the concepts of bitterness and unpleasantness seem to be related semantically (i.e., unpleasant events are sometimes referred to as "bitter").

The relative similarity/dissimilarity of the mixture components may also be considered as an important contributor to the interactive effects of sucrose and quinine. Sucrose and quinine are rated as very dissimilar (Frank et al., 1991), and sweet and bitter stimuli are very seldom confused (unpublished observations). It follows that the distinct sensations elicited by a mixture of sucrose and quinine would not lend themselves to conceptual integration. However, it seems unlikely that the perceptual similarity of sucrose and quinine accounts entirely for the suppression of bitterness by sucrose observed in the present and past studies (Bartoshuk, 1975; Kamen et al., 1961; Lawless, 1979). Lawless (1979) has suggested that the mutual suppression of sweetness and bitterness in sucrosequinine mixtures might be mediated at the sensory coding level. This would mean that the intensity of the mixture components percepts would be reduced prior to the initiation of judgment processes. Since the suppression is prior to judgment, the conceptual manipulations of Experiment $2 B$ would have no effect on the magnitude of suppression across the four experimental conditions. If this interpretation of the sucrose-quinine findings is correct, it suggests that the manipulation of instructions and possibly other judgmental variables can be used to distinguish between interactions that are prior to judgment and those that occur as part of the judgment process.

\section{GENERAL DISCUSSION}

Experiments designed to evaluate interactions in tasteodor and taste-taste mixtures have used many different scaling procedures to assess the intensities of various mixture components. Subjects have been asked to judge the intensity of a mixture's taste or odor without reference to a particular quality label (Enns \& Hornung, 1985; Hornung \& Enns, 1984; Murphy \& Cain, 1980; Murphy, Cain, \& Bartoshuk, 1977). They have also rated the intensity of a single target taste quality (Frank \& Byram, 1988; Kamen et al., 1961). In other experiments, subjects have made estimates of total mixture intensity which were then broken down into ratings of multiple-component intensities (Bartoshuk, 1975; Lawless, 1982; Smith \& McBurney, 1969), or they made independent intensity ratings for multiple attributes of the mixtures (Gillan, 1983; Kuznicki \& Ashbaugh, 1979; Lawless, 1979; Schifferstein \& Frijters, 1990). Little attention has been given to the consequences of these variations in procedure. Experiments 1 and 2 of the present report provide evidence that seemingly minor procedural changes involving the manner in which subjects judge mixture stimuli can have a significant impact on the results of mixture experiments.

When subjects judged only the sweetness or bitterness of the mixture stimuli, sweetness and bitterness enhancement was observed for some mixtures. Using similar procedures, other investigators have also found odor- and taste-induced enhancement of bitter and sweet. For example, Kamen et al. (1961) reported that the bitterness of caffeine was enhanced by citric acid when subjects were asked to judge only the bitterness of caffeine-citric acid solutions. There also appears to be evidence for citralinduced enhancement of sweetness in an experiment by Murphy and Cain (1980) (see their Figure 4). In the condition where the apparent odor-induced taste enhancement occurs, subjects judged only the intensity of the taste component of the taste-odor mixture. Murphy and Cain found no evidence for citral-induced enhancement of the taste of sodium chloride, but the taste of sucrose (presumably sweet) appears to be enhanced by citral at the higher concentrations of sucrose. The effect of citral on sweetness is small, as would be predicted on the basis of the effects of lemon odorant on sweetness (see Figure 2 above). Finally, previous research in our laboratory (Frank \& Byram, 1988; Frank et al., 1989; Frank et al., 1990) has demonstrated that strawberry odor enhances the sweetness of sucrose when only the sweetness of the strawberrysucrose mixtures is rated.

Mixture-induced taste quality enhancement is reduced or eliminated when appropriate attribute rating scales are provided. This was demonstrated in Experiments 1 and 2, and is also supported by the findings of other investigators. Gillan (1983) had subjects make simultaneous magnitude estimates for the sweet, salty, sour, bitter, lemon, and licorice qualities of taste-taste and taste-odor mixtures, and found suppression of both the specific tastes and the specific odors in the mixtures. Total-intensity judgments were broken down into five ratings (sweet, salty, sour, bitter, and other) in an experiment by Bartoshuk (1975). She reported that hydrochloric acid $(\mathrm{HCl}$, a primarily sour stimulus) suppressed the bitterness of quinine hydrochloride $(\mathrm{QHCl})$ in $\mathrm{HCl}-\mathrm{QHCl}$ mixtures.

Taken together, the available evidence supports the speculation that odor- or taste-induced enhancement of specific taste qualities in mixtures of chemosensory stimuli is strongly influenced by the instructions given to subjects and the specific taste and odor qualities of a mixture. When one or few stimulus attributes are rated, subjects exhibit a tendency to combine quality dimensions of the stimuli that are either perceptually or conceptually similar. As subjects are provided with additional, appropriate rating categories, either no interaction or mixture suppression replaces enhancement. Suppression or no interaction is also observed when the quality components of the mixture are dissimilar. Empirical support for the idea that component similarity can influence mixture 
interactions was obtained by Frank et al. (1991). These investigators reported that the amount of enhancement or suppression of taste observed for mixtures of six odorants with sucrose, sodium chloride, and citric acid was highly correlated with ratings of the similarity between the unmixed tastant and odorant.

One might be tempted to conclude that mixture experiments should always use multiple, simultaneous, independent ratings of "appropriate" sensory qualities in order to avoid the "biases" observed with the other ratings procedures. In our view, this would be a mistake. The observations made in the various conditions of Experiments 1 and 2 may have important applications under different circumstances. For example, under the normal conditions of coffee consumption, how do the odors and tastes of the coffee interact? Under these circumstances, the coffee drinker is typically asked, "Is your coffee too sweet?" or, "Is your coffee too bitter?" These questions are analogous to those asked in the sweetness-only and bitternessonly conditions of Experiments 1 and 2, and therefore the responses of subjects asked about only the sweetness or bitterness of a stimulus may predict the responses of people to food products more accurately than when subjects make simultaneous ratings of multiple stimulus attributes. The results of a recent, unpublished experiment from our laboratory reinforce this view. When subjects were asked to match the sweetness of a strawberry-sucrose mixture to the sweetness of an unmixed sucrose solution, they chose a sucrose solution that was significantly more concentrated than the actual concentration of sucrose in the mixture. Thus, the odor-induced enhancement of sweetness observed in Experiment 1 was predictive of the outcome of a sweetness-matching experiment. Rather than labeling one finding or another as artifactual, thereby impuning its significance, it is our view that psychophysicists should evaluate how perceptual and judgmental factors influence responses to multiple-quality mixtures. This approach will yield a richer, more generalized description of mixture effects than will one that relies on a single, privileged method of intensity scaling.

\section{REFERENCES}

ANDERSON, N. H. (1981). Foundations of information integration theory. New York: Academic Press.

Baktoshuk, L. M. (1975). Taste mixtures: Is suppression related to compression? Physiology \& Behavior, 14, 643-649.

EnNs, M. P., HoRnung, D. E. (1985). Contributions of smell and taste to overall intensity. Chemical Senses, 10, 357-366,

Frank, R. A., \& ARChambo, G. (1986). Intensity and hedonic judgments of taste mixtures: An information integration analysis. Chemical Senses, 11, 427-438.

Frank, R. A., \& Byram, J. (1988). Taste-smell interactions are tastant and odorant dependent. Chemical Senses, 13, 445-455.
Frank, R. A., Ducheny, K., \& Mize, S. J. S. (1989). Strawberty odor, but not red color, enhances the sweetness of sucrose solutions. Chemical Senses, 14, 371-377.

Frank, R. A., Shaffer, G., \& Smith, D. V. (1991). Taste-odor similarities predict taste enhancement and suppression in taste-odor mixtures. Chemical Senses, 16, 523.

Frank, R. A., Wessel, N., \& Shaffer, G. (1990). The enhancement of sweetness by strawberry odor is instruction-dependent. Chemical Senses, 15, 576.

GillaN, D. J. (1983). Taste-taste, odor-odor, and taste-odor mixtures: Greater suppression within than between modalities. Perception \& Psychophysics, 33, 183-185.

Gregson, R. A. M., BaKer, A.F.H. (1973). Sourness and bitterness: Confusions over sequences of taste judgments. British Journal of Psychology, 64, 71-76.

Hornung, D. E., \& ENns, M. P. (1984). The independence and integration of olfaction and taste. Chemical Senses, 9, 97-106.

Kamen, J. M., Pilgrim, F. J., Gutman, N. J., Kroll, B. J. (1961). Interactions of suprathreshold taste stimuli. Journal of Experimental Psychology, 62, 348-356.

Kuznicki, J. T., Ashbaugh, N. (1979). Taste quality differences within the sweet and salty taste categories. Sensory Processes, 3 , 157-182.

LAWLESS, H. T. (1977). The pleasantness of mixtures in taste and olfaction. Sensory Processes, 1, 227-237.

LAWLESS, H. T. (1979). Evidence for neural inhibition in bittersweet taste mixtures. Journal of Comparative \& Physiological Psychology, 93, 538-547.

LAWLESs, H. T., (1982). Paradoxical adaptation to taste mixtures. Physiology \& Behavior, 25, 149-152.

Lawless, H. T., Glatter, S., \& Hohn, C. (1991). Context-dependent changes in the perception of odor quality. Chemical Senses, 16, 349-360.

MURPHY, C., \& CAIN, W. S. (1980). Taste and olfaction: Independence vs. interaction. Physiology \& Behavior, 24, 601-605.

Murphy, C., Cain, W. S., Bartoshuk, L. M. (1977). Mutual action of taste and olfaction. Sensory Processes, 1, 204-211.

O'MAHonY, M. (1991). Descriptive analysis and concept alignment. In H. T. Lawless \& B. P. Klein (Eds.), Sensory science theory and applications in foods (pp. 223-267). New York: Marcel Dekker.

OMAhony, M., Goldenberg, M., STedmon, J., \& Alford, J. (1979). Confusion in the use of the taste adjectives "sour" and "bitter." Chemical Senses \& Flavor, 4, 301-318.

Parducci, A. (1965). Category judgment: A range-frequency model. Psychological Review, 72, 407-418.

SChifferstein, H. N. J., \& Fruters, J. E. R. (1990). Sensory integration in citric acid/sucrose mixtures. Chemical Senses, 15, 87-109.

Shaffer, G., Frank, R. A. (1990). An investigation of taste-smell interactions across four tastants and six odorants. Chemical Senses, $15,638$.

SmITH, D. V., M MCBuRNeY, D. H. (1969). Gustatory cross-adaptation: Does a single mechanism code the salty taste? Journal of Experimental Psychology, 80, 101-105.

\section{NOTE}

1. Heterogeneous taste-quality mixtures refer to mixtures of two tastants which elicit two distinct taste-quality sensations (e.g., the mixing of moderately intense concentrations of sucrose and sodium chloride results in a solution that is judged to be both sweet and salty).

(Manuscript received July 22, 1992; revision accepted for publication February 10, 1993.) 